\title{
Truncated Epithelial Sodium Channel $\beta$ Subunit Responsible for Liddle Syndrome in a Chinese Family
}

\author{
Peng Fan ${ }^{a}$ Chao-Xia Lu ${ }^{b}$ Kun-Qi Yang ${ }^{a}$ Pei-Pei Lu ${ }^{a}$ Su-Fang Hao ${ }^{a}$ \\ Fang Luo ${ }^{a}$ Hui-Min Zhang ${ }^{a}$ Lei Song ${ }^{a}$ Hai-Ying Wu ${ }^{a}$ Jun Cai ${ }^{a}$ \\ Xue Zhang $^{\mathrm{b}}$ Xian-Liang Zhou $^{\mathrm{a}}$ \\ aDepartment of Cardiology, Fuwai Hospital, National Center for Cardiovascular Diseases, \\ Chinese Academy of Medical Sciences and Peking Union Medical College, Beijing, China; \\ ${ }^{b}$ McKusick-Zhang Center for Genetic Medicine, State Key Laboratory of Medical Molecular \\ Biology, Institute of Basic Medical Sciences, Chinese Academy of Medical Sciences and \\ Peking Union Medical College, Beijing, China
}

\section{Keywords}

Liddle syndrome · Epithelial sodium channel · Frameshift mutation · Phenotype ·

Genetic testing

\begin{abstract}
Background/Aims: Liddle syndrome (LS) is a rare autosomal dominant disease caused by mutations in genes coding for epithelial sodium channel $(\mathrm{ENaC})$ subunits. The aim of this study was to identify the mutation responsible for the LS in an extended Chinese family. Methods: DNA samples from the proband with early-onset, treatment-resistant hypertension, and hypokalemia and 19 additional relatives were all sequenced for mutations in exon 13 of the $\beta$-ENaC and $\gamma$-ENaC genes, using amplification by polymerase chain reaction and direct DNA sequencing. Results: Genetic testing of exon 13 of SCNN1B revealed duplication of guanine into a string of 3 guanines located at codon 602. This frameshift mutation is predicted to generate a premature stop codon at position 607 , resulting in truncated $\beta$-ENaC lacking the remaining 34 amino acids, including the crucial PY motif. Among a total of 9 participants with the identical mutation, different phenotypes were identified. Tailored treatment with amiloride was safe and effective in alleviating disease symptoms in LS. No mutation of SCNN1G was identified in any of the examined participants. Conclusions: We report here a family affected by LS harboring a frameshift mutation (c.1806dupG) with a premature stop codon deleting the
\end{abstract}

P.F. and C.-X.L. contributed equally to this work.

Xue Zhang

McKusick-Zhang Center for Genetic Medicine

State Key Laboratory of Medical Molecular Biology

Institute of Basic Medical Sciences, Chinese Academy of Medical Sciences

School of Basic Medicine Peking Union Medical College

5 Dong Dan San Tiao, Beijing 100005 (China)

E-Mail xuezhang@pumc.edu.cn
Xian-Liang Zhou

Department of Cardiology, Fuwai Hospital

National Center for Cardiovascular Diseases

Chinese Academy of Medical Sciences and

Peking Union Medical College

No. 167, Beilishi Road, Beijing 100037 (China)

E-Mail zhouxianliang0326@ hotmail.com 


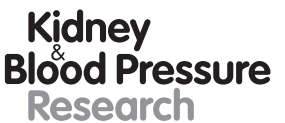

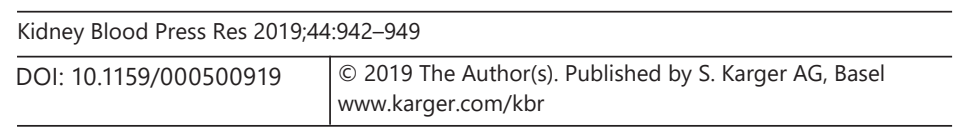

Fan et al.: Truncated ENaC $\beta$ Subunit in LS

PY motif of $\beta$-ENaC. Our study demonstrates that the earlier LS patients are diagnosed by genetic testing and treated with tailored medication, the greater the likelihood of preventing or minimizing complications in the vasculature and target organs. (c) 2019 The Author(s)

\section{Introduction}

Elevated blood pressure (BP) is currently the single largest contributor to the global burden of disease and to global mortality [1]. In contrast to the case in essential hypertension, in only a minority of patients with secondary hypertension, an underlying hereditary cause is responsible for the elevated BP [2]. Monogenic hypertension syndromes are a particular subset of secondary hypertension, involving hypertensive disorders that follow Mendelian inheritance patterns due to causative single-gene mutations [3].

Liddle syndrome (LS, OMIM \#177200) is an uncommon and autosomal dominant form of monogenic hypertension which was first described by Liddle et al. [4]. Its typical clinical characteristics include salt-sensitive hypertension with early penetrance, hypokalemia, suppression of both plasma renin and aldosterone secretion, and metabolic alkalosis. Whereas the presentation of LS varies somewhat even within families carrying the same mutation. The effective treatment includes a low-salt diet and epithelial sodium channel (ENaC) inhibitors, such as amiloride and triamterene, but not spironolactone therapy [5]. ENaC, composed of $\alpha$, $\beta$, and $\gamma$ subunits, plays an important role in BP control by regulating of sodium and water reabsorption [6]. SCNN1A, SCNN1B, and SCNN1G each encode one of the $3 \mathrm{ENaC}$ subunits [7]. Gain-of-function mutations in these genes lead to LS [8-10]. Clinically, many patients with LS are misdiagnosed and develop severe complications at an early age, due to the absence of genetic evidence that supports the diagnosis of LS [11]. Genetic testing, regarding as a rapid and accurate tool, is highly advisable for patients with a clinical suspicion of LS.

In this study, we characterized a Chinese family with LS, caused by a frameshift mutation (c.1806dupG) in $S C N N 1 B$, which generated a new stop codon at position 607. Genotype phenotype analysis showed heterogeneity among the family members, especially in terms of the existence of hypokalemia. Genetic diagnosis and tailored medicine were beneficial for combating LS, avoiding a series of severe complications associated with malignant hypertension and hypokalemia.

\section{Methods}

\section{Subjects}

The proband (III-16), a 35-year-old male, was hospitalized at the hypertension center of Fuwai Hospital, Beijing, China, for the suspicion of LS due to early-onset and treatmentresistant hypertension, spontaneous and persistent hypokalemia, and low plasma renin concentration (PRC). A total of 19 at-risk family members (Fig. 1) of the proband agreed to undergo clinical evaluation, biochemical examination, and genetic testing.

\section{Clinical and Biochemical Evaluation}

The proband underwent echocardiography; computed tomography (CT) of the kidneys, adrenal glands, renal arteries, coronary artery, and aorta; and cardiac magnetic resonance imaging during hospitalization. After maintaining a standing position for $2 \mathrm{~h}$, all participants underwent biochemical measurement of PRC and plasma aldosterone concentration (PAC) at Fuwai Hospital by chemiluminescence immunoassay using the LIAISON ${ }^{\circledR}$ Direct 


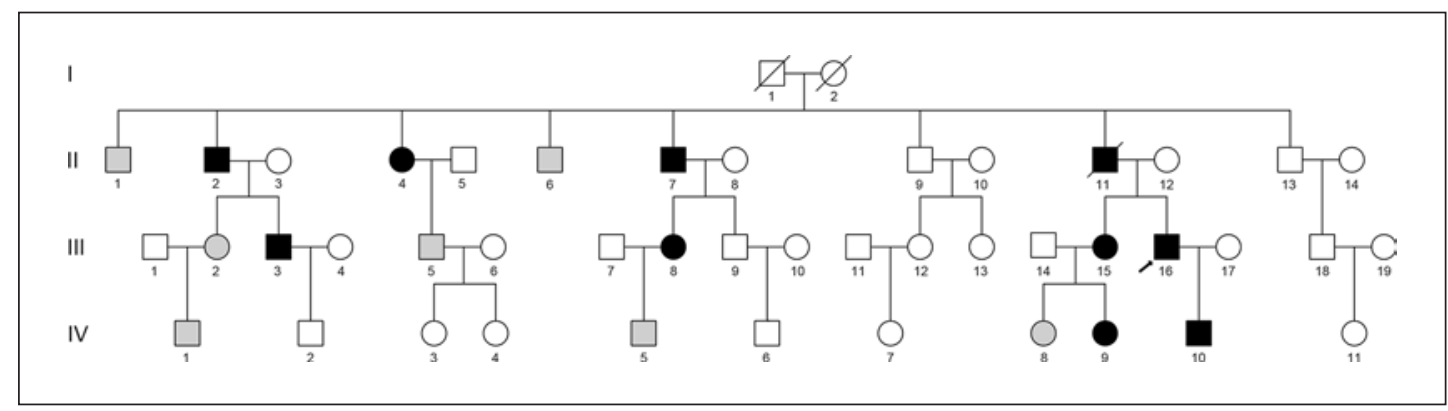

Fig. 1. Family pedigree. Those with the identified mutation are shown as black symbols; those without the mutation are shown as gray symbols; deceased individuals are shown as symbols with a diagonal line; the arrow indicates the proband.

Renin kit (DiaSorin S.p.A, Vercelli, Italy) and the LIAISON ${ }^{\circledR}$ Aldosterone kit (DiaSorin Inc., Stillwater, MN, USA). Other biochemical examinations were performed using standard methods.

\section{Genetic Testing}

Sequencing of exon 13 of both $S C N N 1 B$ and $S C N N 1 G$ is highly advisable in patients with early-onset hypertension [12]. Genomic DNA was extracted from peripheral blood leucocytes using the QIA Amp DNA Blood Mini kit (QIAGEN, Hilden, Germany) in accordance with standard protocols. SCNN1B (GenBank accession number NM_000336) and SCNN1G (GenBank accession number NM_001039) are closely associated with LS [13]. Apart from $\alpha$-ENaC C479R [14], all of the other mutations are clustered within exon 13 of either SCNN1B or SCNN1G [15]. Amplification of exon 13 of $S C N N 1 B$ and $S C N N 1 G$ was conducted by polymerase chain reaction using 2 gene-specific primer pairs, in accordance with the work of Yang et al. [11] ( $\beta$-subunit primer pair: 5'-CAAGAATCACCTCCCAGGAAGC-3', 5'-GTGATTCTACAGAGCCCTTGGC-3'); $\gamma$-subunit primer pair (5'-CTTGGGAATCAGGGTTCCTGTG-3', $5^{\prime}$-AAGCAGGCTTTTTGGTCAGAGT-3'). All polymerase chain reaction products were sequenced by an ABI Prism 377 DNA sequencer (Applied Biosystems, Foster City, CA, USA). To confirm the mutations, the exons were sequenced in the forward and reverse directions twice.

\section{Results}

\section{Clinical Features}

In the proband, high BP (160/120 mm Hg) had been detected in 1997 at the age of 15, during a routine physical examination, although no further intervention was performed. By November 2008, the symptoms of hemoptysis and fatigue had led the patient to undergo a medical examination, which revealed hypertension. The patient's serum potassium levels fluctuated between 2.40 and $3.50 \mathrm{mmol} / \mathrm{L}$ in the past 3 years before 2017, despite the oral administration of potassium chloride. His BP was also poorly controlled, ranging from 140 to $220 \mathrm{~mm} \mathrm{Hg}$ (systolic) and from 100 to $150 \mathrm{~mm} \mathrm{Hg}$ (diastolic), despite the administration of amlodipine, nifedipine, spirolactone, or losartan. He had also experienced dizziness in 2015 and 2016. In September 2017, the proband was admitted to Fuwai Hospital for further evaluation of hypertension and hypokalemia.

Biochemical examination showed a low serum potassium level of $3.21 \mathrm{mmol} / \mathrm{L}$, low PRC (1.2; reference value, 2.8-39.9 $\mu \mathrm{IU} / \mathrm{mL}$ ), and normal PAC (4.3; reference value, 3.0-23.6 ng/ 
Table 1. Clinical and biochemical features of all participants in this family

\begin{tabular}{|c|c|c|c|c|c|c|c|c|c|}
\hline \multirow[t]{2}{*}{ Subjects } & \multirow{2}{*}{$\begin{array}{l}\text { Age, } \\
\text { years }\end{array}$} & \multirow{2}{*}{$\begin{array}{l}\text { Onset age } \\
\text { of hyper- } \\
\text { tension, } \\
\text { years }\end{array}$} & \multirow{2}{*}{$\begin{array}{l}\mathrm{BP} \\
\mathrm{mm} \mathrm{Hg}\end{array}$} & \multirow{2}{*}{$\begin{array}{l}\text { Serum } \mathrm{K}^{+} \text {, } \\
\mathrm{mmol} / \mathrm{L}\end{array}$} & \multirow{2}{*}{$\begin{array}{l}\mathrm{PRC}^{\mathrm{a}}, \\
\mu \mathrm{IU} / \mathrm{mL}\end{array}$} & \multirow{2}{*}{$\begin{array}{l}\mathrm{PAC}^{\mathrm{a}} \\
\mathrm{ng} / \mathrm{dL}\end{array}$} & \multirow{2}{*}{$\begin{array}{l}\text { Amiloride* } \\
\text { dosage/other } \\
\text { antihyper- } \\
\text { tensives }\end{array}$} & \multicolumn{2}{|l|}{ Follow-up } \\
\hline & & & & & & & & $\begin{array}{l}\mathrm{BP} \\
\mathrm{mm} \mathrm{Hg}\end{array}$ & $\begin{array}{l}\text { serum } \mathrm{K}^{+} \\
\mathrm{mmol} / \mathrm{L}\end{array}$ \\
\hline \multicolumn{10}{|l|}{ Affected } \\
\hline II-2 & 73 & 48 & $220 / 120$ & 4.80 & 149.3 & 3.0 & $2.5 \mathrm{mg} /-$ & $140 / 80$ & 5.07 \\
\hline II-4 & 70 & 25 & $200 / 130$ & 4.38 & 8.1 & 2.8 & $2.5 \mathrm{mg} /-$ & $120 / 80$ & 4.50 \\
\hline II-7 & 66 & 36 & $200 / 140$ & 3.40 & 37.2 & 3.3 & $2.5 \mathrm{mg} /-$ & $123 / 75$ & 4.11 \\
\hline III-3 & 35 & 19 & $180 / 160$ & 3.57 & 6.4 & 2.1 & $5.0 \mathrm{mg} /-$ & $140 / 100$ & 5.44 \\
\hline III-8 & 35 & 16 & $180 / 108$ & 4.30 & 103.1 & 4.4 & $2.5 \mathrm{mg} /-$ & $120 / 90$ & 4.84 \\
\hline III-15 & 37 & 16 & $180 / 110$ & 3.91 & 3.0 & 1.8 & $5.0 \mathrm{mg} / \mathrm{ARB}$ & $125 / 96$ & 5.11 \\
\hline III-16 & 35 & 15 & $220 / 150$ & 2.40 & 10.5 & 4.9 & $2.5 \mathrm{mg} /-$ & $126 / 88$ & 5.30 \\
\hline IV-9 & 3 & 3 & $130 / 80$ & 4.33 & 100.2 & 3.0 & $-/-$ & $-/-$ & - \\
\hline IV-10 & 10 & 3 & $150 / 130$ & 2.80 & $<0.5$ & 1.9 & $2.5 \mathrm{mg} /-$ & $100 / 70$ & 4.38 \\
\hline \multicolumn{10}{|c|}{ Unaffected $^{\#}$} \\
\hline II-9 & 62 & 50 & $173 / 90$ & 5.10 & 34.0 & 11.4 & $-/-$ & $-/-$ & - \\
\hline II-13 & 53 & - & $116 / 80$ & 4.84 & 52.6 & 9.2 & $-1-$ & $-/-$ & - \\
\hline III-9 & 30 & - & $124 / 72$ & 4.82 & 34.8 & 5.1 & $-1-$ & $-/-$ & - \\
\hline III-12 & 32 & - & $127 / 77$ & 4.40 & 61.5 & 19.8 & $-1-$ & $-1-$ & - \\
\hline III-13 & 30 & - & $124 / 90$ & 5.18 & 51.6 & 33.8 & $-1-$ & $-1-$ & - \\
\hline III-18 & 29 & - & $132 / 85$ & 4.45 & 54.8 & 10.6 & $-/-$ & $-/-$ & - \\
\hline IV-2 & 10 & - & $102 / 63$ & - & 45.9 & 9.7 & $-/-$ & $-1-$ & - \\
\hline IV-3 & 18 & - & $115 / 76$ & 4.46 & 2.7 & 2.4 & $-1-$ & $-/-$ & - \\
\hline IV-4 & 12 & - & $131 / 73$ & 4.46 & 23 & 15.6 & $-/-$ & $-1-$ & - \\
\hline IV-6 & 6 & - & $98 / 65$ & 5.09 & 84.7 & 11.2 & $-1-$ & $-1-$ & - \\
\hline IV-7 & 4 & - & $74 / 49$ & 4.24 & 128.2 & 16.8 & $-/-$ & $-/-$ & - \\
\hline
\end{tabular}

$\mathrm{BP}$, blood pressure; serum $\mathrm{K}^{+}$: reference value, 3.5-5.3 mmol/L; PRC, plasma renin concentration: reference value, 4.4-46.1 $\mu \mathrm{IU} / \mathrm{mL}$; PAC, plasma aldosterone concentration: reference value, 3.0-35.3 ng/dL; ARB, angiotensin receptor blocker. \# Unaffected: the unaffected subjects had no identified mutation. * Compound amiloride containing amiloride (2.5 mg) and hydrochlorothiazide (25 mg) in each tablet. ${ }^{a}$ PRA and PRC examination after keeping upright position for $2 \mathrm{~h}$.

$\mathrm{dL}$ ) in a standing position and significantly increased N-terminal prohormone of brain natriuretic peptide (826.3; reference value, lower than $150 \mathrm{pg} / \mathrm{mL}$ ). Microalbuminuria and adrenocorticotrophic hormone levels were normal. Echocardiography, aortic CT, and cardiac magnetic resonance suggested right coronary valve prolapse, severe aortic valve insufficiency, and enlargement of the ascending aorta and left heart (left atrium anteroposterior diameter $49 \mathrm{~mm}$ and left ventricle end diastolic diameter $74 \mathrm{~mm}$, in echocardiography). Because of these severe complications of LS, the proband had to receive an aortic valve replacement. After this procedure, coronary artery, renal, and adrenal abnormalities were ruled out by a CT scan.

Across 3 generations of the proband's family, 10 individuals among 20 participants had a history of hypertension. Most of them were early-onset hypertensive patients $(7 / 10)$ younger than 30 years old, although the total age range was 3-50 years old. The early penetrance of LS was revealed in the proband's son (IV-10), in whom hypertension (150/130 mm $\mathrm{Hg}$ ) emerged as 3 years of age and hypokalemia accompanied by fatigue emerged at 8 years of age. This boy had only been treated with potassium chloride, through which no significant improvement in fatigue or serum potassium level was achieved. The proband's father (II-11) had first become aware of his own hypertension due to suffering from severe epistaxis at the age of 29 years. Ten years later, he died of stroke with uncontrolled BP, despite the adminis- 
Fig. 2. Sanger sequencing chromatogram reveals a heterozygous frameshift mutation at codon position 603 of exon 13 of SCNN1B.

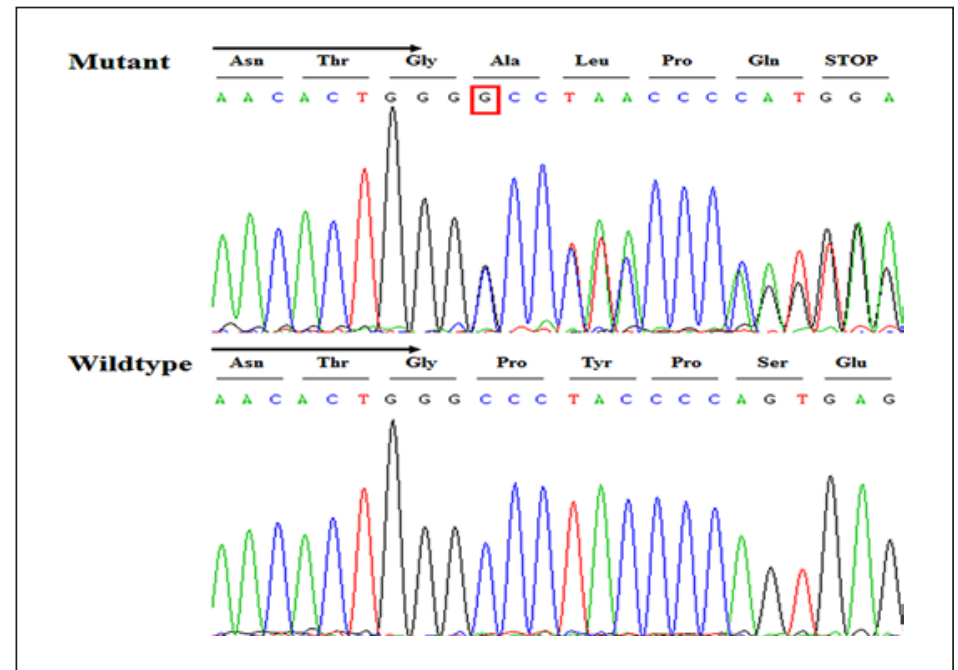

tration of amlodipine. Similarly, individual II-7 had also suffered from stroke because of hypertension at the age of 60 years. Three members of the family (II-7, III-16, and IV-10) were identified to have low serum potassium concentration. Suppressed PRC and PAC were noted in individuals III-15, IV-3, and IV-10. Only PAC was suppressed in individuals II-2, II-4, III-3, and IV-9 (Table 1).

\section{Genetic Analysis}

As shown in Figure 2, a heterozygous frameshift mutation in exon 13 of SCNN1B was detected in the proband. This involved the insertion of an additional guanine into a sequence of 3 adjacent guanines at codon 603 (c.1806dupG). This frameshift mutation transformed P603, Y604, P605, and S606 into A603, L604, P605, and Q606, as well as producing a new stop codon at position 607, deleting the last 34 amino acids of $\beta$-ENaC, including its PY motif. There were 9 affected patients with this causative mutation, while the other 11 participants lacked it in this family (Table 1). There was no mutation in sequenced exon 13 of SCNN1G in 20 participants in this family.

\section{Tailored Medicine for Mutation Carriers}

As a first treatment option, each hypertensive patient carrying the identified mutation was advised to adopt a healthy lifestyle, such as consuming a low-salt diet, quitting smoking and alcohol, and performing a moderate amount of exercise. In China, only compound amiloride containing amiloride $(2.5 \mathrm{mg}$ ) and hydrochlorothiazide (25 $\mathrm{mg})$ in each tablet are available. Among 9 mutation carriers diagnosed with LS, 8 accepted treatments with compound amiloride; the exception was individual IV-9, whose mother refused treatment for her. Initially, $8 \mathrm{LS}$ patients took one compound amiloride every day for a month and then reexamined the BP and electrolytes (Table 1). Subjects III-3 and III-15 showed normokalemia, but their BP was still uncontrolled higher than 160/110 $\mathrm{mm}$ Hg. Hence, 2 tablets compound amiloride each day was adjusted for them. The rest of the 6 patients showed significant improvement in decreasing their BP and normalizing their potassium levels. After dose adjustment, subjects III-15 showed normal BP (125/96 $\mathrm{mm} \mathrm{Hg}$ ), while subjects III-3 also needed an angiotensin receptor blocker to keep BP lower than 140/100 mm Hg. 


\section{Kidney \\ Blood Pressure \\ Research}

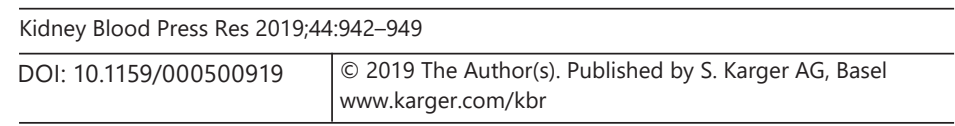

Fan et al.: Truncated ENaC $\beta$ Subunit in LS

\section{Discussion}

This study characterizes a frameshift mutation of the $\beta$ subunit of $\mathrm{ENaC}$, generating a premature stop codon at position 607 and deleting the remaining 34 amino acids. Specifically, genotype - phenotype analysis indicated the diagnosis of LS in a Chinese family with this mutation. Underestimation of the prevalence and variable phenotypes in LS remind us of the importance of genetic testing for patients with a suspected LS. Tailored medicine with ENaC inhibitor improved symptoms and prognosis for LS patients specifically and effectively.

With the progression of research on LS, the pathogenesis has been clarified. ENaC activity is enhanced with increasing probability of the channel being open and with the increasing number of channels expressed at the apical membrane of principal cells [16, 17], leading to greater $\mathrm{Na}^{+}$reabsorption and plasma volume expansion, as well as a consequent increase in BP and decreases in renin and aldosterone secretion and hypokalemic metabolic alkalosis, resulting from a transepithelial lumen-negative voltage generated by increased $\mathrm{Na}^{+}$reabsorption, driving $\mathrm{K}^{+}$secretion across the apical membrane [8]. Both $S C N N 1 B$ and SCNN1G mutations mainly influence the PY motif, which acts as the binding site of a specific ubiquitin ligase (Nedd4-2) and interacts with Nedd4-2 to catalyze the ubiquitination of ENaCs, resulting in their internalization and degradation $[17,18]$. It is believed that the PY motif is essential for interaction with cytoskeletal proteins, endocytosis of the channel, or both [19]. SCNN1B and SCNN1G mutations associated with LS are incapable of combining with Nedd4-2 because of the deletion or alteration of the PY motif, but can activate ENaC [20,21].

Types of mutations in $S C N N 1 B$ include frameshift, nonsense, and missense mutations [15]. To date, 24 SCNN1B mutations associated with LS have been identified. SCNN1B is thus regarded as the gene most commonly associated with LS pathogenicity. Most LS mutations in $S C N N 1 B$ are missense mutations located in exon 13, which alter the PY motif [12]. Thus far, among all reported $S C N N 1 B$ mutations, 9 frameshift mutations associated with LS have been identified. In 2017, Cui et al. [22] reported 2 isolated LS patients from different families who carried the SCNN1B mutation (c.1806_1807insG), although adequate genetic evidence on the mutated genes in their family members could not be obtained. The current study characterized this heterozygous mutation in SCNN1B (c.1806dupG) in a pedigree containing 9 LS patients. The proband's hypertensive father most probably carried the identified mutation, and that the proband inherited this condition from his father, since the proband's father (II-11) had died of stroke resulting from uncontrolled hypertension and his mother (II-12) had not.

Genotype was consistent with the phenotype in the aspect of resistant and early-onset hypertension. Nine carriers with identified pathogenic mutation had resistant hypertension and were diagnosed with LS. In addition, the age of onset of hypertension in 9 affected patients ranged from 3 to 48 years. Owing to early penetrance, the age of onset of LS is generally before 30 years [12]. Unusually, participant II-2 with LS was identified to have hypertension at the age of 48 , which was the first time he had undergone a general physical examination. Hence, we supposed that he might have been hypertensive but without any symptoms before the diagnosis at this late age. On the other hand, there were also late-onset patients with LS $[12,23]$.

However, as in other monogenic disorders, phenotypic variability is a notable characteristic of LS. Namely, the same mutation can result in different phenotypes, even in one family, including heterogeneity in the clinical manifestations [24]. For example, in the case of LS, hypokalemia may differ in its severity within the same family or may not be present at all [25]. In this family with LS, the serum potassium level varied among 9 carriers. Indi- 


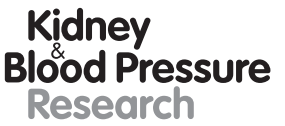

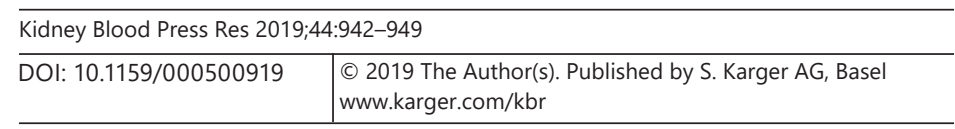

Fan et al.: Truncated ENaC $\beta$ Subunit in LS

viduals II-7, III-16, and IV-10 manifested typical hypokalemia with LS, while 4 carriers were detected with a normal serum potassium level because they had taken medicines for many years, namely, potassium chloride by III-3 and III-8 and antihypertensive agents $\left(\mathrm{Ca}^{2+}\right.$ channel blockers) by II- 2 and II- 4 . In contrast, carriers III- 15 and IV- 9 were not hypokalemia altogether. This is consistent with the observation in the previous study in which the LS was identified that hypokalemia is not a universal finding among affected individuals [26].

The ENaC inhibitors such as amiloride are safe and effective for controlling BP and correcting hypokalemia in LS patients. These drugs are known to act directly upon ENaC, as showed by their ability to extinguish sodium flux through the channel when expressed in Xenopus oocytes [19]. The dosage of compound amiloride varied among the LS patients in this family, indicating that they had different responses to amiloride [24]. However, uncontrolled BP was shown in III-3, even upon the administration of amiloride, hydrochlorothiazide, and angiotensin receptor blocker. Hence, besides medications, a healthy lifestyle is the basic option for treating LS, such as a low-salt diet and moderate exercise. Moreover, overstimulation of $\mathrm{ENaC}$ by metabolic abnormalities in obese individuals appears to be a probable cause of the hypertension [27]. Therefore, individual III-3 should consider losing weight for controlling BP due to a high body mass index $\left(27.5 \mathrm{~kg} / \mathrm{m}^{2}\right)$.

Genetic testing is an accurate and convenient tool for LS diagnosis. Previous studies suggested that genetic testing should be performed in patients with early-onset and lowrenin hypertension [12] or in early-onset hypertension patients after primary aldosteronism and renal and renovascular diseases have been ruled out [13]. In consideration of the variability in phenotypes and age of onset of hypertension, genetic diagnosis by sequencing exon 13 of SCNN1B and SCNN1G should be first considered for patients with hypertension resistant to conventional treatment. Of course, we should certainly pay particular attention to hypertensives with hypokalemia, with suppressed PRC or PAC and a notable family history of earlyonset hypertension. If there is no mutation in $S C N N 1 B$ and $S C N N 1 G$ in patients with clinical suspicion of LS, SCNN1A is worthy of sequencing lastly [14].

\section{Conclusion}

We report a frameshift mutation in SCNN1B in a Chinese family with LS. This pathogenic mutation deletes the PY motif by generating a premature stop codon. This work enriches the range of genetic evidence for $L S$, indicating that genetic testing should be strongly recommended for patients with a clinical suspicion of LS, especially if there is a family history of hypertension, thereby guiding accurate diagnosis and early treatment facilitating a better prognosis.

\section{Statement of the Ethics}

This study was approved by the Ethics Committee of Fuwai Hospital. All participants provided written informed consent.

\section{Disclosure Statement}

The authors declare that they have no competing interests. 


\begin{tabular}{l|l}
\hline \multicolumn{2}{l}{ Kidney Blood Press Res 2019;44:942-949 } \\
\hline DOI: 10.1159/000500919 & $\begin{array}{l}\text { (c) 2019 The Author(s). Published by S. Karger AG, Basel } \\
\text { www.karger.com/kbr }\end{array}$ \\
\hline
\end{tabular}

Fan et al.: Truncated ENaC $\beta$ Subunit in LS

\section{Funding Sources}

This work was supported by CAMS Innovation Fund for Medical Sciences (2016-I2M-1002), the National Key Research and Development Program of China (2016YFC1300100), National Natural Science Foundation of China (81600305 and 81400187), Beijing Nova Program (Z171100001117026), and PUMC Graduate Innovation Fund (2018-1002-01-14).

\section{References}

1 Poulter NR, Prabhakaran D, Caulfield M. Hypertension. Lancet. 2015 Aug;386(9995):801-12.

2 Ott C, Schneider MP, Schmieder RE: Ruling out secondary causes of hypertension. EuroIntervention. 2013 May; 9 Suppl R:R21-8.

3 Ahn SY, Gupta C. Genetic Programming of Hypertension. Front Pediatr. 2018 Jan; 5:285.

4 Liddle GW, Bledsoe T, Coppage WS Jr. A familial renal disorder simulating primary aldosteronism but with negligible aldosterone secretion. Trans Assoc Am Physicians. 1963;76:199-213.

5 Tetti M, Monticone S, Burrello J, Matarazzo P, Veglio F, Pasini B, et al. Liddle Syndrome: Review of the Literature and Description of a New Case. Int J Mol Sci. 2018 Mar;19(3):1-15.

6 Rossier BC. Epithelial sodium channel (ENaC) and the control of blood pressure. Curr Opin Pharmacol. 2014 Apr; 15:33-46.

7 Hanukoglu I, Hanukoglu A. Epithelial sodium channel (ENaC) family: Phylogeny, structure-function, tissue distribution, and associated inherited diseases. Gene. 2016 Apr;579(2):95-132.

8 Rossier BC, Staub 0, Hummler E. Genetic dissection of sodium and potassium transport along the aldosteronesensitive distal nephron: importance in the control of blood pressure and hypertension. FEBS Lett. 2013 Jun; 587(13):1929-41.

9 Shimkets RA, Warnock DG, Bositis CM, Nelson-Williams C, Hansson JH, Schambelan M, et al. Liddle's syndrome: heritable human hypertension caused by mutations in the beta subunit of the epithelial sodium channel. Cell. 1994 Nov;79(3):407-14.

10 Hansson JH, Nelson-Williams C, Suzuki H, Schild L, Shimkets R, Lu Y, et al. Hypertension caused by a truncated epithelial sodium channel gamma subunit: genetic heterogeneity of Liddle syndrome. Nat Genet. 1995 Sep;11(1): 76-82.

11 Yang KQ, Lu CX, Xiao Y, Liu YX, Jiang XJ, Zhang X, et al. A novel frameshift mutation of epithelial sodium channel $\beta$-subunit leads to Liddle syndrome in an isolated case. Clin Endocrinol (Oxf). 2015 Apr;82(4):611-4.

12 Liu K, Qin F, Sun X, Zhang Y, Wang J, Wu Y, et al. Analysis of the genes involved in Mendelian forms of low-renin hypertension in Chinese early-onset hypertensive patients. J Hypertens. 2018 Mar;36(3):502-9.

13 Yang KQ, Lu CX, Fan P, Zhang Y, Meng X, Dong XQ, et al. Genetic screening of SCNN1B and SCNN1G genes in earlyonset hypertensive patients helps to identify Liddle syndrome. Clin Exp Hypertens. 2018;40(2):107-11.

14 Salih M, Gautschi I, van Bemmelen MX, Di Benedetto M, Brooks AS, Lugtenberg D, et al. A Missense Mutation in the Extracellular Domain of $\alpha \mathrm{ENaC}$ Causes Liddle Syndrome. J Am Soc Nephrol. 2017 Nov;28(11):3291-9.

15 Pagani L, Diekmann Y, Sazzini M, De Fanti S, Rondinelli M, Farnetti E, et al. Three Reportedly Unrelated Families With Liddle Syndrome Inherited From a Common Ancestor. Hypertension. 2018 Feb;71(2):273-9.

16 Kellenberger S, Schild L. International Union of Basic and Clinical Pharmacology. XCI. structure, function, and pharmacology of acid-sensing ion channels and the epithelial Na+ channel. Pharmacol Rev. 2015;67(1):1-35.

17 Palmer LG, Patel A, Frindt G. Regulation and dysregulation of epithelial Na+ channels. Clin Exp Nephrol. 2012 Feb; 16(1):35-43.

18 Schild L. The epithelial sodium channel and the control of sodium balance. Biochim Biophys Acta. 2010 Dec; 1802(12):1159-65.

19 Snyder PM, Price MP, McDonald FJ, Adams CM, Volk KA, Zeiher BG, et al. Mechanism by which Liddle's syndrome mutations increase activity of a human epithelial Na+ channel. Cell. 1995 Dec;83(6):969-78.

20 Rizzo F, Staub 0. NEDD4-2 and salt-sensitive hypertension. Curr Opin Nephrol Hypertens. 2015 Mar;24(2):111-6.

21 Ronzaud C, Staub O. Ubiquitylation and control of renal Na+ balance and blood pressure. Physiology (Bethesda). 2014 Jan;29(1):16-26.

22 Cui Y, Tong A, Jiang J, Wang F, Li C. Liddle syndrome: clinical and genetic profiles. J Clin Hypertens (Greenwich). 2017 May; 19(5):524-9.

23 Kyuma M, Ura N, Torii T, Takeuchi H, Takizawa H, Kitamura K, et al. A family with liddle's syndrome caused by a mutation in the beta subunit of the epithelial sodium channel. Clin Exp Hypertens. 2001 Aug;23(6):471-8.

24 Gong L, Chen J, Shao L, Song W, Hui R, Wang Y. Phenotype-genotype analysis in two Chinese families with Liddle syndrome. Mol Biol Rep. 2014 Mar;41(3):1569-75.

25 Bogdanović R, Kuburović V, Stajić N, Mughal SS, Hilger A, Ninić S, et al. Liddle syndrome in a Serbian family and literature review of underlying mutations. Eur J Pediatr. 2012 Mar;171(3):471-8.

26 Botero-Velez M, Curtis JJ, Warnock DG. Brief report: liddle's syndrome revisited-a disorder of sodium reabsorption in the distal tubule. N Engl J Med. 1994 Jan;330(3):178-81.

27 Bubien JK. Epithelial Na+ channel (ENaC), hormones, and hypertension. J Biol Chem. 2010 Jul;285(31):23527-31. 